\title{
SMARTTANK: AN INTERNET-OF-THINGS (IOT) APPLICATION TO AUTOMATE THE WATER TANK REFILLING USING COMPUTER VISION AND AI
}

\author{
Henry Hamilton ${ }^{1}$, Yu Sun ${ }^{2}$, Fangyan Zhang ${ }^{3}$ \\ ${ }^{1}$ Capistrano Valley High School, CA 91765, USA \\ ${ }^{2}$ California State Polytechnic University, Pomona, CA, 91768, USA \\ ${ }^{3}$ ASML, San Jose, CA, 95131, USA
}

\begin{abstract}
This system provides a method of automatically keeping water bowls full and refilling every time it is detected that they are not. This is highly useful for anyone who owns a pet, as it decreases the amount of work the owner will need to do. The system uses an AI model, trained with over a thousand images of water bowls. This allows it to accurately determine when a bowl needs filling. When an empty bowl is spotted, a subsystem consisting of a valve and other electronic parts releases stored water into the bowl. Through experimentation it has been shown the accuracy of the system is about $97 \%$ under optimal lighting conditions. Without a light source, the system does not function. Currently, the components are not of the highest quality and the system only works with the bowl used in testing. There are future plans to train the model with new pictures featuring an assortment of bowls. Additionally, an LED could be added to the system to solve the issue of it not working without external light.
\end{abstract}

\section{KEYWORDS}

Artificial Intelligence, image detection, RPI system processor

\section{INTRODUCTION}

Pet owners face a variety of difficulties on a daily basis, which is expected, as they are responsible for maintaining the life of another organism. For the most part, these difficulties are specific to what type of animal you own, although there are a few that are universal. One of the more problematic of these is providing your pet drinking water while you are not physically present. Whether it be a vacation or business trip, for a week or for a month, your pet still needs fresh water to drink. Some animals, namely rodents, have specialized bottles which do not require constant attention, but most use water bowls. Keeping these bowls full may be a matter of life or death. Even if the situation is not so grave, needing to regularly complete this task is tedious and unenjoyable.

There are a few systems currently available that can accomplish a similar purpose, though all have limitations [1-5]. As mentioned earlier, an existing method is a rodent water bottle [6]. This involves a bottle held upside down with a short metal tube leading towards the rodent. The tube's width decreases as it moves downward. Normally, the end of the tube is sealed with a small rubber ball, held in place by gravity. When the rodent is thirsty, it can use its snout to push up the rubber ball into the wider section of the tube, leading to the flow of water around it as the seal is David C. Wyld et al. (Eds): MLNLP, BDIoT, ITCCMA, CSITY, DTMN, AIFZ, SIGPRO - 2020 pp. 129-136, 2020. CS \& IT - CSCP 2020 
broken. While simplistic yet efficient, the problem lies in the fact that it cannot be used by other animals. It dispenses water at an extremely slow rate, making it useless for larger animals. Additionally, many animals cannot push up the ball, or even comprehend how the system functions. The other solution in existence is a larger water bowl with a button that when pressed, pumps out water from a tank nearby [7]. This solution is limited by the strength required to push the button. Only larger animals, like dogs or cats can utilize this tool. Another assumption made is that the animal in question can even equate an action like pushing a button with being able to drink water. For most reptiles, this is nearly impossible.

My tool involves a raised platform supported by legs and a base. A water bowl can be placed under the platform. The base serves two purposes: firstly, it provides stability to the platform and prevents it from falling over. The second purpose is that it provides a clearly defined location to place the bowl, so that it is within the view of the camera. The platform uses a camera to firstly identify the bowl, then decide whether the bowl is empty or full of water. If the bowl is empty, a valve residing on the platform will open, and water from a nearby tank will flow through it. The water is then channeled into the bowl. The process occurs every 30 minutes, thus autonomously filling the bowl and satisfying whatever animal depends on it without human intervention. An additional feature is that it shuts off at night and turns back on in the morning. This provides a multitude of advantages. Firstly, the tool doesn't work under low light conditions, so being active at the specified times brings no advantages. Additionally, without sunlight, the water will not evaporate at a noticeable rate, so refilling the bowl isn't necessary at night. Finally, by shutting it off energy can be saved.

Two experiments were done to prove the effectiveness of the solution. In the first experiment, the AI was subjected to 60 pictures, broken down into the 3 categories "Filled" "Partially Filled" and "Empty". It sorted the images and accuracy was recorded. These three categories are important, as the AI will most likely encounter all 3 during usage. The second experiment tested accuracy in different light levels with a constant water level. The bowl remained completely filled while the brightness of the room's light was adjusted. Once again, 60 pictures were tested this time under 3 light levels. Results showed that darkness and empty bowls caused the most confusion, but accuracy was still acceptably high in all cases.

The rest of the paper is ordered in this fashion: Section 2 describes a number of obstacles met when attempting to design a solution to the problem; Section 3 provides a detailed overview on how the solution works and what components are in play, along with how the challenges noted in Section 2 were overcome; Section 4 provides information regarding experiments done to prove the solution's effectiveness, while Section 5 presents some related works. Section 6 concludes the paper and predicts the project's future. It also provides a few examples regarding how to solve issues listed earlier in the paper.

\section{Challenges}

Throughout the system development, we ran into several challenges that needed overcoming. Here is a brief overview of some of the most difficult challenges that we faced when developing this application.

\subsection{Challenge 1: Differentiate between Filled and Empty Bowls}

When the light levels are too low, the AI has trouble differentiating between filled and empty bowls. This was due to a variety of causes. Initially, when training the AI, all pictures used were taken during midday in a brightly illuminated room. The model was therefore unable to recognize 
objects in a different environment. An attempt was made to remedy this by taking pictures during the morning and using those for training. There was very limited success to this though, since the camera equipment was not of the highest quality and therefore look blurrier photos in the absence of light. The final aspect to this issue was that filled and empty bowls looked very similar from above. Even when a bowl is empty, the small amount of water lining the bottom reflects light just like a full bowl would. Since the camera is directly above the bowl looking down into it, the two were hard to differentiate. Darkness only made this harder.

\subsection{Challenge 2: Proper Camera Position}

Positioning the camera correctly was a challenge. In the initial design, there was no raised platform and the camera was haphazardly taped to a table. This was obviously an issue, since the camera was not secure and instead wobbled around. Additionally, the height of the table meant that the bowl would be far away from the camera, leaving an undesirable amount of space around the bowl. These issues were fixed when the platform was implemented. Now the camera was fixed to a specific spot and could no longer move. The platform was not too high, so the bowl was more clearly visible. Finally, the base helped keep the bowl position consistent, so it would always remain the pictures center. The only remaining challenge would be to remove the extraneous space around the bowl. This was done by zooming in slightly.

\subsection{Challenge 3: Water Control System}

While the AI posed the most difficulty, the water control system was no easy feat either. The setup was simple initially, involving a tube, a tank and a valve. Unfortunately, one important aspect was overlooked: water doesn't just flow on its own. There was no force to propel the water out of the tank and through the tubing, so even when the valve was open water would not flow. A solution was devised where a tire pump would be used to pressurize the water, and it worked somewhat, but with limited success. The other more obvious solution would be to use gravity. This led to more consistent results, but the water pressure was still unfavorably low.

\section{Solution}

My project is a system whose purpose is to refill a bowl of water once it is detected that the bowl is no longer full. It is made up of multiple components that work in conjunction towards this goal. The first is the software. The system uses an AI model trained on a vast amount of bowl pictures with varying water levels. When an image, captured with an RPI camera, is sent to the AI, it returns whether the bowl is considered full or empty, along with a confidence level. Both of these values are key in determining the next action. If the AI's confidence exceeds 0.65 , the result returned will be used. However, if the AI's confidence is below the previously specified number, the system considers the bowl's state to be the one not returned by the AI, e.i., if the AI returns "Full" the rest of the system will run as if the bowl was empty. The rest of the software side of the system contains some code which activates a pin on the RPI if the bowl is believed to be empty. A series of hardware components open a valve once a signal from the pin is received, which eventually results in water pouring into the bowl from a nearby storage tank. A general list of components involved in the completion of goals are:

- A trained AI model to determine water level in bowls

- Additional software to take pictures and take actions in accordance with the AI's decision

- Multiple pieces of hardware that control the valve.

- Water tank in the form of a milk jug.

- RPI camera to capture bowl photos.

- A wood and plastic stand which acts as a base for all the components. 


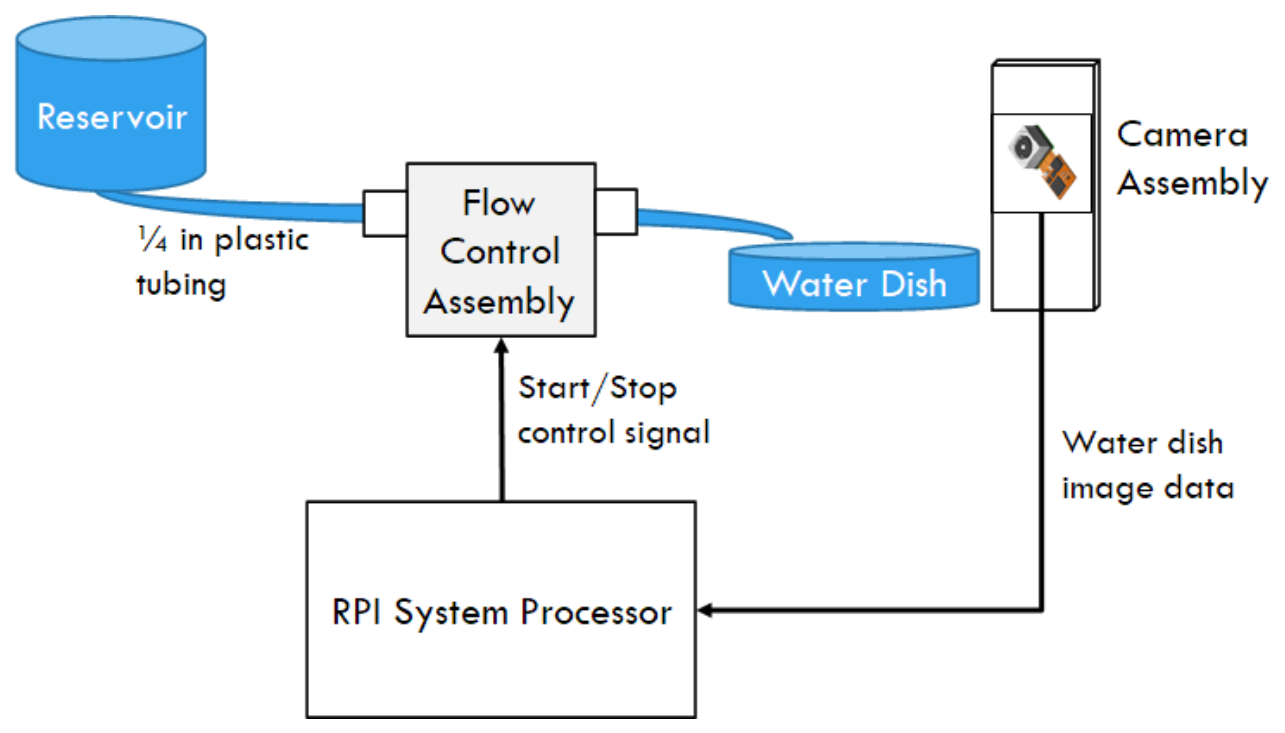

Figure 1: Overview of the components

The first component to be noted is the trained AI model. This was created using Google Cloud's Vision API and was trained with over 1500 images. These images were an assortment of filled, partially filled and empty bowls with varying light levels. To collect these photos, a short program was written that would continuously take pictures of a bowl throughout an entire day. As the day progressed, the movement of the sun would slightly change the light level of each picture in order to make the pictures unique. This was done over multiple days, each day using a different water level. The completed model was then downloaded onto an RPI. An RPI camera attachment was purchased online and also added to the RPI. The code works rather simply. First, a picture is captured. Next, the AI determines whether the bowl is filled or empty and the confidence level. Finally, a function is used to activate a pin on the RPI if the bowl is empty. The next component to be addressed is the valve control system. The electrical components for it are pictured below. The pins on the RPI are linked with a MOSFET switch.

Before further information on the switch, it must be noted that the solenoid valve requires $15 \mathrm{~V}$ to function correctly. More specifically, it remains closed as long as it receives a constant $15 \mathrm{~V}$. The RPI cannot supply $15 \mathrm{~V}$, as its maximum is $5 \mathrm{~V}$, so the power supply is used to that end. When the RPI sends $5 \mathrm{~V}$ to the switch, it breaks the connection between power supply and valve, resulting in the valve opening to allow water flow. The water is stored in a milk jug at higher elevation than the rest of the system. This is important, because otherwise the water does not have enough pressure to flow. The water is transported through plastic tubing, eventually emptying into the water bowl. The above-mentioned steps occur multiple times per day at 30 second intervals. The system is deactivated at night since the camera does not operate without light. It should be noted that for each cycle, only a small amount of water is released. To fill an entire bowl the system must be left on for close to an hour.

A four-legged platform is the body of the project, and on it sits all the electrical components. It is made of a flat board filled with holes, 4 PVC pipe legs and 4 white wooden 1 x 2 in planks that surround the bottom of the legs, forming a base. The top wooden platform provides a surface for the RPI and other components, but its other purpose is to elevate the camera to the optimal height. In order for the AI to best make decisions regarding the bowl, it must take a picture with only the bowl within view. The camera is attached to the bottom on the wooden platform, and points straight down at the bowl. At a height of 18 in, the camera perfectly captures the bowl with 
few spaces around it. The wood planks around the legs provide stability and also wall off a square area directly under the platform for the bowl.

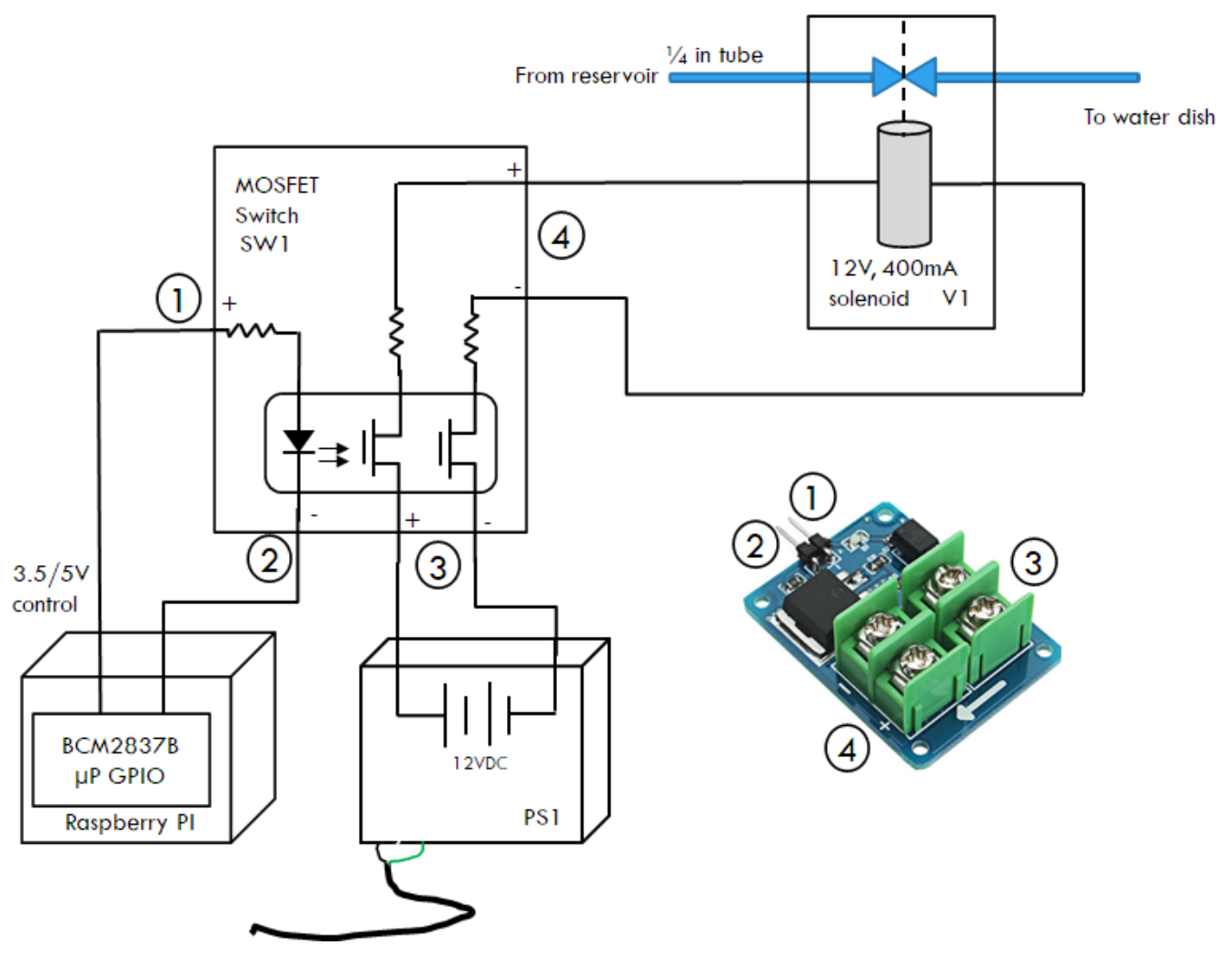

Figure 2: Details of the components

\section{EXPERIMENT}

The first experiment's goal is to test the AI model's accuracy under 3 different conditions (Table 1). The three conditions are "Completely Full", "Half Full" and "Empty". For each condition, 30 trials were done in order to obtain statistically significant results. All other factors were held constant. This testing was done in a brightly lit room during the day, so the bowl was exposed to both natural and artificial light. The bowl was positioned directly under the camera to avoid interference from other factors. On the results table, T shows that the AI's deduction was correct, or true, while $\mathrm{F}$ shows it was incorrect, or false.

Table 1: AI model's accuracy under 3 different conditions

\begin{tabular}{|l|l|r|}
\hline Condition & \multicolumn{1}{|c|}{ Results } & Accuracy \\
\hline $\begin{array}{l}\text { Completely } \\
\text { Full }\end{array}$ & $\begin{array}{l}\text { T, T, T, T, T, T, T, T, T, T, T, T, T, T, T, T, T, T, T, T, T, T, T, T, T, } \\
\text { T, T, T, T, T }\end{array}$ & $100 \%$ \\
\hline Half Full & $\begin{array}{l}\text { T, T, T, T, T, T, T, T, T, T, T, T, T, T, T, T, T, T, T, T, F, T, T, T, F, } \\
\text { T, T, T, T, T }\end{array}$ & $93 \%$ \\
\hline Empty & $\begin{array}{l}\text { F, T, T, T, T, T, T, T, T, T, T, T, T, T, T, T, T, T, T, T, T, T, T, T, T, } \\
\text { T, T, T, F, T }\end{array}$ & $93 \%$ \\
\hline
\end{tabular}




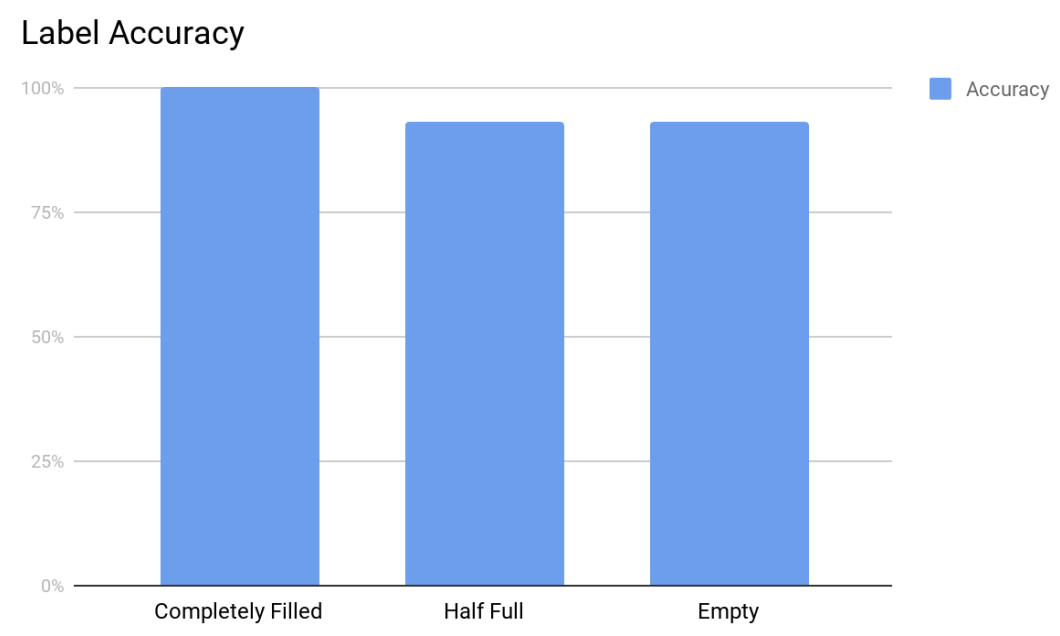

Figure 3: Label Accuracy in bright room

Accuracy appears to be higher for full bowls than empty bowls. This is further made clear by the confidence intervals for each result. On average, the AI had a confidence of about 0.7 for full bowls, only 0.6 for empty bowls and half full bowls.

This experiment's goal is to test the AI's accuracy under multiple light levels (Table 2). There are three levels tested in the experiment, one with light in the room completely off, one with the light at half brightness and one with the light at full brightness. The lights in question are LED lamps and the curtains are closed, making sunlight negligible. Water level was held at a constant level of completely filled. 30 trials were done for each light level. $\mathrm{T}$ shows that the deduction was correct, while F shows it was incorrect.

Table 2: AI's accuracy under multiple light levels

\begin{tabular}{|l|l|r|}
\hline Condition & \multicolumn{1}{|c|}{ Results } & Accuracy \\
\hline $\begin{array}{l}\text { Light at Full } \\
\text { Strength }\end{array}$ & $\begin{array}{l}\text { T, T, T, T, T, T, T, T, T, T, T, T, T, T, F, T, T, T, T, T, T, F, T, T, } \\
\text { F, T, T, F, T }\end{array}$ & $84 \%$ \\
\hline $\begin{array}{l}\text { Light at Half } \\
\text { Strength }\end{array}$ & $\begin{array}{l}\text { T, T, T, T, T, T, T, T, T, T, T, T, T, T, T, T, T, T, T, T, T, T, T, T, } \\
\text { T, T, T, T, T, T }\end{array}$ & $100 \%$ \\
\hline Dark & $\begin{array}{l}\text { F, F, T, F, T, F, F, T, F, F, T, F, T, F, F, F, T, T, F, T, T, F, T, F, T, } \\
\text { T, T, T, F, T, T, F, F }\end{array}$ & $47 \%$ \\
\hline
\end{tabular}

The results in this experiment were slightly unexpected. While a dark room showed a great decrease in accuracy, a brightly lit room led to a drop in accuracy as well. The reasoning behind this could be that most of the training pictures were done in a room with natural lighting from the outside, as opposed to a bright artificial light, hence the AI better classifies a dimmer room more similar to a naturally lit one. 
Labels

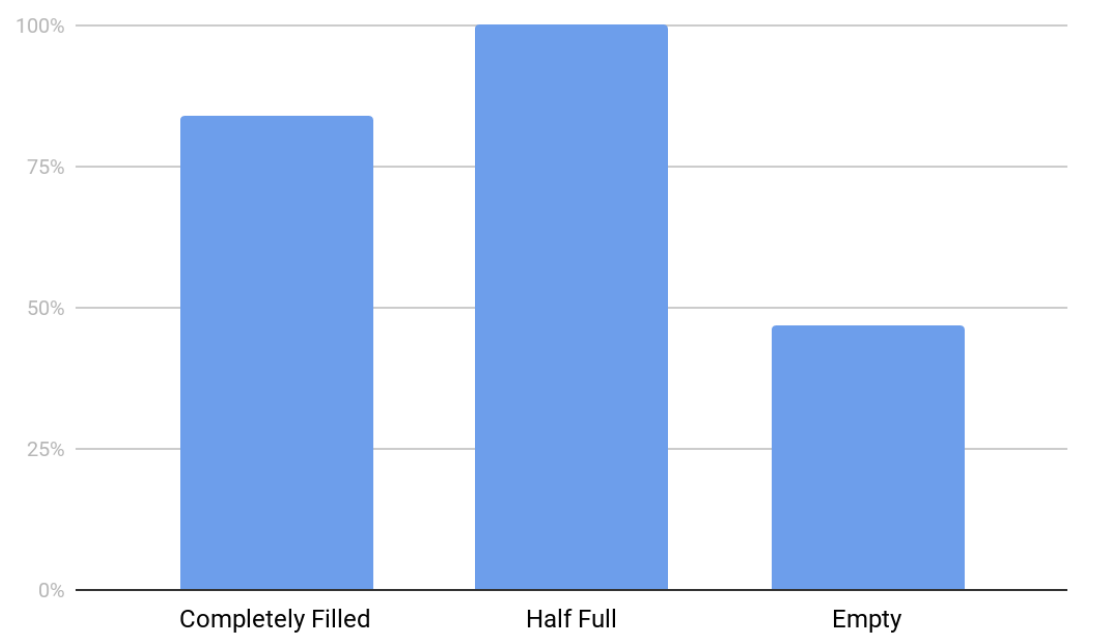

Figure 4: Label Accuracy in dark room

The experiments confirmed a few notions that were previously held. Firstly, having the system deactivated during night hours is a good idea, since it clearly does not work without light. Conversely, however, too much light has a negative impact on accuracy as well. The optimal place for the system would likely be outdoors in a shaded area or indoors but only exposed to ambient light. The expectation is that this system would be able to be used with most animals, indoors or outdoors. To this end it seems operational, and just requires careful positioning when initially set up. On the subject of water level, it appears to perform decently well at all water levels, even though confidence might be lower when the bowl is not full. The lack of confidence, however, does not appear to greatly impact performance.

\section{RELATED WORK}

Automatic water dispenser for pets is an automatically refilling pet bowl that flushes the water and refills it daily in accordance with a timer. My tool is similar as it is used for an identical purpose, refilling water bowls. Norris' tool [9] is superior in the aspect that it not only refills but also drains existing water to always maintain fresh, clean water in the bowl. The downside with Norris' tool is that it will refill water at each time interval even if there is water still in the bowl. This leads to water being wasted.

A Cloud Image Classification for Thrash Collecting LEGO Mindstorms EV3 Robot that uses Google Cloud's Vision API [10-11] to identify plastic waste. Additionally, it is able to identify trash bins and differentiate between trash bin types to correctly dispose of collected trash. Both my project and this one-use Vision to train our AI models, though the difference lies in that this project utilizes it for two functions: identifying waste and identifying trash cans. The prototype shown in the report appears to mainly use color to easily identify trash cans, rather than the symbols that are used in public areas. Compared to my own project, this one's AI is most likely simpler and possesses fewer images, as color identification is far easier than identifying water level.

International Journal of Landscape Architecture Research uses Google Vision API to detect landmarks and capture the visual character of landscapes. For example, the model would be able 
to determine whether a picture of a landscape contains forests, or the ocean, or a city etc. This project's similarity with mine lies that it also uses Google Vision API to derive data from a picture. The difference lies in that this AI uses 320 labels compared to my 2 labels. Additionally, because of the number of labels, along with the complexity of the pictures entered, the number of training images far exceeds mine. Overall, this project shows the potential of Google Vision.

\section{CONCLUSION AND FUTURE WORK}

My project utilizes AutoML Vision to train an AI model that can identify the water level of a bowl. This is combined with a system that opens and closes a valve, which is able to then fill said bowl when the water level is detected to be low. Together these components provide a method for refilling water bowls for pets autonomously.

Currently the method, while functional, is not the most efficient way to accomplish the task. Its accuracy decreases heavily when there is not sufficient lighting. The completed project is bulky and difficult to transport. It takes up a fair amount of space, making it unsuitable for smaller animal enclosures. However, its supports also prevent larger animals from accessing the bowl. Additionally, the supports may encourage animals to climb on it, which would damage it or the animal. Finally, it depends heavily on the bowl being the same in all aspects except for the water. If the bowl is moved off center, or if something is placed within the bowl, the AI will cease to function correctly. Using a different bowl leads to similar issues.

A few solutions have been thought up to combat the current issues. An LED could be installed onto the raised platform, so that it illuminates the bowl, solving the lighting issue. A plastic shell could be used to cover the sensitive components, so climbing animals will not damage it. The legs and base of the platform could be altered to allow animals to access the bowl more easily. A possible solution could be to remove the base and configure the legs to be at an angle.

\section{REFERENCES}

[1] Poffenroth, Kevin. "Animal drinking water supply apparatus." U.S. Patent 5,452,683 issued September 26, 1995.

[2] Ewell, Anthony S. "Automatic pet food dispenser." U.S. Patent 5,433,171 issued July 18, 1995.

[3] Krishnamurthy, S. "Automatic pet waterer." U.S. Patent 6,928,954 issued August 16, 2005.

[4] King, Wayne. "Automatic waterbowl for pets." U.S. Patent 6,253,709 issued July 3, 2001.

[5] Graves, James. "Endless water bowl." U.S. Patent Application 10/367,019 filed July 28, 2005.

[6] Honeycutt, Jennifer A., Jenny QT Nguyen, Amanda C. Kentner, and Heather C. Brenhouse. "Effects of water bottle materials and filtration on Bisphenol A content in laboratory animal drinking water." Journal of the American Association for Laboratory Animal Science 56, no. 3 (2017): 269-272.

[7] Sexton, James E. "Pet food dish elevating assembly." U.S. Patent 5,584,263, issued December 17, 1996.

[8] Olde, Jarl Rune. "Automatic water dispenser." U.S. Patent 3,868,926, issued March 4, 1975.

[9] Norris, J. (2003). U.S. Patent Application No. 10/426,865.

[10] BENLİAY, A., \& ALTUNTAŞ, A. (2019). Visual Landscape Assessment with the Use of Cloud Vision API: Antalya Case. International Journal of Landscape Architecture Research (IJLAR) EISSN: 2602-4322, 3(1), 07-14.

[11] Othman, Z., Abdullah, N. A., Chin, K. Y., Shahrin, F. F. W., Ahmad, S. S., \& Kasmin, F. (2018). Comparison on Cloud Image Classification for Thrash Collecting LEGO Mindstorms EV3 Robot. International Journal of Human and Technology Interaction (IJHaTI), 2(1), 29-34.

(C) 2020 By AIRCC Publishing Corporation. This article is published under the Creative Commons Attribution (CC BY) license. 